\title{
Stability analysis of the $D$-dimensional nonlinear Schrödinger equation with trap and two- and three-body interactions
}

\author{
A. Gammal ${ }^{1}$, T. Frederico ${ }^{2}$, Lauro Tomio ${ }^{1}$, and F.Kh. Abdullaev ${ }^{1 *}$ \\ ${ }^{1}$ Instituto de Física Teórica, Universidade Estadual Paulista, \\ 01405-900 São Paulo, Brazil \\ ${ }^{2}$ Departamento de Fúsica, Instituto Tecnológico da Aeronáutica, \\ Centro Técnico Aeroespacial, 12228-900 São José dos Campos, SP, Brazil
}

(November 6, 2018)

\begin{abstract}
Considering the static solutions of the $D$-dimensional nonlinear Schrödinger equation with trap and attractive two-body interactions, the existence of stable solutions is limited to a maximum critical number of particles, when $D \geq 2$. In case $D=2$, we compare the variational approach with the exact numerical calculations. We show that, the addition of a positive three-body interaction allows stable solutions beyond the critical number. In this case, we also introduce a dynamical analysis of the conditions for the collapse.
\end{abstract}

PACS: 03.75.Fi; 47.20.Ky; 02.30.Jr; 31.75.Pf

Keywords: Nonlinear Schrödinger Equation; trapped two and three-body atomic systems; multidimensional systems

Recent experiments on Bose Einstein Condensation (BEC) [1] have brought great attention to its theoretical formulation. Atomic traps are effectively described by the Ginzburg-Pitaevskii-Gross (GPG) formulation of the nonlinear Schrödinger equation (NLSE) [2], which includes two-body interaction. When the atoms have negative two-body scattering lengths, a formula for the critical maximum number of atoms was presented in ref. [3]. In ref. [4 6], the formulation was extended in order to include the effective potential originated from the threebody interaction. In this case, in three-dimensions, it was shown that a kind of first order phase-transition occurs. In this connection, as also considered in the motivations given in [5, 6], it is relevant to observe that recently it was reported the possibility of altering continuously the twobody scattering length, from positive to negative values, by means of an external magnetic field [7]. Within such perspective, the two-body binding energy can be close to zero, and one can approach the so-called Efimov limit, which corresponds to an increasing number of three-body bound states 8. Near this limit, nontrivial consequences can occur in the dynamics of the condensate, such that one should also consider three-body effects in the effective nonlinear potential.

In the present work, we study the critical number of atoms in arbitrary $D$-dimensions, using a variational procedure; and also by an exact numerical approach in the case of dimension $D=2$. The $D$-dimensional NLSE, with attractive two-body interactions, was previously analyzed in models of plasma and light waves in nonlinear media [9]. The collapse conditions, in this case, were investigated without [10] and with [11] the harmonic potential term. In case of $D=3$, it was shown that a repulsive nonlinear three-body interaction term can extend considerably the critical limit for the existence of stable solutions 4 [6].

Motivated by the observed high interest in stable solutions for arbitrary $D$, we look for variational solutions in a few significant cases $(D=1,2,4$ and 5$)$ not previously considered, when a three-body interaction term, parametrized by $\lambda_{3}$, is added to the effective non-linear interaction that contains a two-body attractive term. Our analysis also shows that, as in case of $D=3$, a kind of first-order phase-transition can occur when $D \geq 4$, for certain cases of $\lambda_{3} \geq 0$. In the present paper, we have also considered the approach given in [12], in order to study the stability conditions in the case of arbitrary $D$, when the non-linear interaction contains two (attractive) and three-body terms.

In order to obtain an analytical approach and verify the validity of the variational Ritz method, we consider in detail the case of $D=2$, with and without the threebody term, comparing the variational results with exact numerical calculations for some relevant physical observables. In this case, we also discuss how the method given in 10] can be extended in order to approach analytically the exact value for the total energy.

By extending the GPG formalism from three to $D$ dimensions, including two 13 and three-body interactions in the effective non-linear potential [5], we obtain

$$
i \hbar \frac{d \psi}{d t}=\left[-\frac{\hbar^{2}}{2 m} \nabla^{2}+\frac{m \omega^{2} r^{2}}{2}+\lambda_{2}|\psi|^{2}+\lambda_{3}|\psi|^{4}\right] \psi,
$$

where $\psi \equiv \psi(\vec{r}, t)$ is the wave-function normalized to the number of atoms $N, \omega$ is the frequency of the trap harmonic potential and $m$ is the mass of the atom. $\lambda_{2}$ and $\lambda_{3}$ are, respectively, the strength of the two- and three-body

*On leaving from Physical Technical Institute, Tashkent, Uzbekistan. 
effective interaction, given in a $D$-dimensional space. $r \equiv|\vec{r}|$ is the hyperradius, such that $\vec{r} \equiv \sum_{i=1}^{D} r_{i} \hat{e}_{i}$ and $\nabla \equiv \sum_{i=1}^{D} \hat{e}_{i} \frac{\partial}{\partial r_{i}}\left(\hat{e}_{i}\right.$ is the unit vector, with $\left.i=1,2, \ldots D\right)$.

The stationary solutions for the chemical potential $\mu$ are given by

$$
i \hbar \frac{d \psi}{d t}=\mu \psi
$$

Considering the general solution of eq.(11), $i \hbar \frac{d \psi}{d t}=\frac{\delta \mathcal{H}}{\delta \psi^{\star}}$, one can obtain the total energy $E$ :

$$
\begin{aligned}
E & =\int d^{D} \vec{r} \mathcal{H}, \text { with } \\
\mathcal{H} & \equiv \frac{\hbar^{2}}{2 m}|\nabla \psi|^{2}+\frac{m \omega^{2} r^{2}}{2}|\psi|^{2}+\frac{\lambda_{2}}{2}|\psi|^{4}+\frac{\lambda_{3}}{3}|\psi|^{6} .
\end{aligned}
$$

Here we consider only attractive two-body interaction, which is more interesting in the case of trapped atoms. For $D=3, \lambda_{2} \equiv-4 \pi \hbar^{2}|a| / m$, where $a$ is the two-body scattering length and $m$ is the mass of the atom. In the case of arbitrary $D, \lambda_{2}$ has dimension of energy times $L^{D}$, where $L$ is a length scale in such space. However, a convenient redefinition of the wave-function in terms of dimensionless variables will absorb this constant, as will be shown.

Our study will be concentrated on the ground state for a spherically symmetric potential. We first consider the case of $\lambda_{3}=0$, using a variational procedure, with a trial Gaussian wave-function for $\psi(\vec{r})$, normalized to $N$, given by

$$
\psi_{v a r}(\vec{r})=\sqrt{N}\left(\frac{1}{\pi \alpha^{2}} \frac{m \omega}{\hbar}\right)^{D / 4} \exp \left[-\frac{r^{2}}{2 \alpha^{2}}\left(\frac{m \omega}{\hbar}\right)\right],
$$

where $\alpha$ is a dimensionless variational parameter. From eq. (3), the corresponding expression for the total variational energy can be expressed as

$$
\begin{aligned}
& E_{v a r}=\hbar \omega \frac{N}{\nu} \mathcal{E}_{v a r}, \\
& \mathcal{E}_{v a r} \equiv \nu\left(\frac{D}{4 \alpha^{2}}+\frac{D \alpha^{2}}{4}\right)-\frac{\nu^{2} \Omega_{D}}{4(2 \pi)^{D / 2} \alpha^{D}}+\frac{G_{3}}{6 \pi^{D}} \frac{\nu^{3} \Omega_{D}^{2}}{3^{D / 2} \alpha^{2 D}},
\end{aligned}
$$

where $\Omega_{D}$ is the solid angle in $D$ dimensions,

$$
\begin{aligned}
& \Omega_{D} \equiv \frac{2 \pi^{D / 2}}{\Gamma(D / 2)}, \quad G_{3} \equiv \frac{\lambda_{3}}{2\left(\lambda_{2}\right)^{2}} \hbar \omega \\
& \text { and } \nu \equiv-\frac{N}{\Omega_{D}} \frac{2 \lambda_{2}}{\hbar \omega}\left(\frac{m \omega}{\hbar}\right)^{D / 2}
\end{aligned}
$$

By using dimensionless variables, $\vec{x} \equiv \sqrt{m \omega / \hbar} \vec{r}$, we redefine the wave-function $\psi$ as

$$
\phi(\vec{x}) \equiv \sqrt{\frac{2\left|\lambda_{2}\right|}{\hbar \omega}} \psi(\vec{r})
$$

such that

$$
\int|\phi(\vec{x})|^{2} d^{D} \vec{x}=N\left[\frac{2\left|\lambda_{2}\right|}{\hbar \omega}\right]\left(\frac{m \omega}{\hbar}\right)^{D / 2}=\nu \Omega_{D} .
$$

The dimensionless equation corresponding to eq. (1), can be rewritten as

$$
\left[\left(-\sum_{1}^{D} \frac{d^{2}}{d x_{i}^{2}}+x_{i}^{2}\right)-|\phi|^{2}+G_{3}|\phi|^{4}-2 \beta\right] \phi=0,
$$

where $\beta \equiv \mu /(\hbar \omega)$ is the dimensionless chemical potential. From eqs. (8) and (4), the trial wave-function can be written as

$$
\phi_{v a r}(x) \equiv \sqrt{\nu \Omega_{D}}\left(\frac{1}{\pi \alpha^{2}}\right)^{D / 4} \exp \left(-\frac{x^{2}}{2 \alpha^{2}}\right),
$$

The variational results, obtained by using the above expressions can be extended analytically to non-integer values of the dimension $D$. Minimization of the energy [eq. (5)], with respect to $\alpha^{2}$, is done numerically by sweeping over $\alpha^{2}$ values. The results for the energy and the chemical potential are shown in Fig. 1. For each value of $D$, one can observe a critical number of atoms, $N_{c}$, related to the critical parameter $\nu_{c}$, only when $D \leq 2$. This critical limit corresponds to the cusps in the upper plot of Fig. 1 and is also observed using exact numerical calculation for $D=3$. It is also interesting to note that for $D>2$ there are two branches of solutions for $\mathcal{E}_{v a r}$ and $\beta$, one stable and the other unstable. In the energy, the lower branch corresponds to stable solutions (minima), while the upper one gives unstable solutions (maxima).

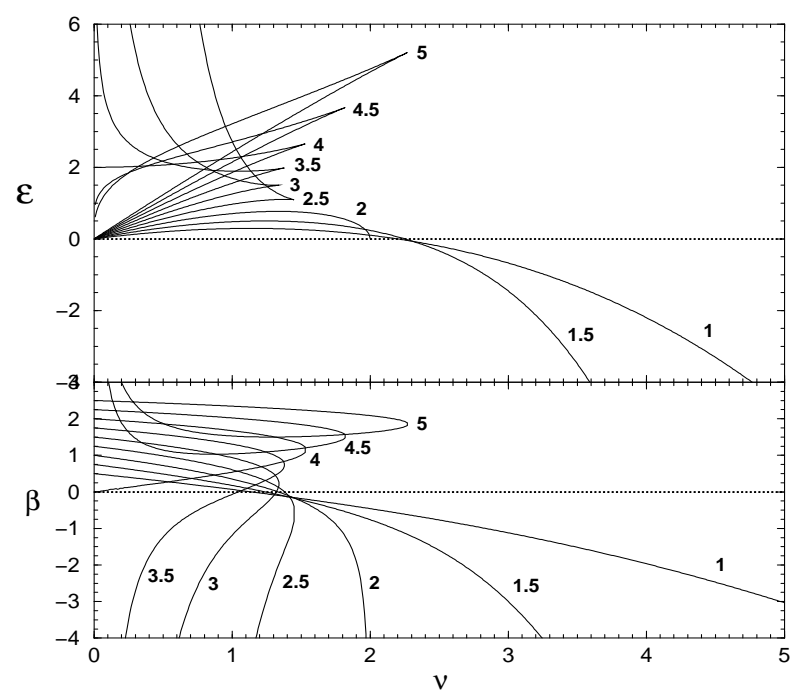

FIG. 1. The variational energy $\mathcal{E}$ (upper part) and chemical potential $\beta$ (lower part), as functions of the reduced number of atoms $\nu$, for several values of the dimension $D$, indicated in each plot. All the quantities are in dimensionless units, as defined in the text. 
The case with $D=2$ is particularly interesting, as no unstable solutions exist and there are stable solutions only for $\nu<2$, such that $\nu_{c}=2$. For $D=2$, the minimization of eq. (5) in respect to $\alpha^{2}$ leads to

$$
\mathcal{E}_{\text {var }}=\nu \sqrt{1-\frac{\nu}{2}}
$$

The behavior of $\nu$, and the corresponding critical limits, as one alters the dimension $D$, has other curious particular results. For example, the critical limit $\nu_{c}$ has a minimum for $D=3\left(\nu_{c}^{(D)} \geq \nu_{c}^{(3)}\right.$ for all $\left.D\right)$.

In conclusion of this part of our work, considering arbitrary $D$ with $\lambda_{3}=0$, there are no stable solutions for eq. (11), if the wave-function $\phi(x)$, given by eq. (9), is normalized to $\nu>\nu_{c}$. Fig. 1 shows that this restriction is strongest for $D=3: \nu_{c}$ is a minimum when compared with $\nu_{c}$ for $D \neq 3$. This is a relevant result, considering that $\nu$ is directly proportional to the number of atoms. Also, it is observed that $\nu_{c}$ increases very fast for $D>3$.

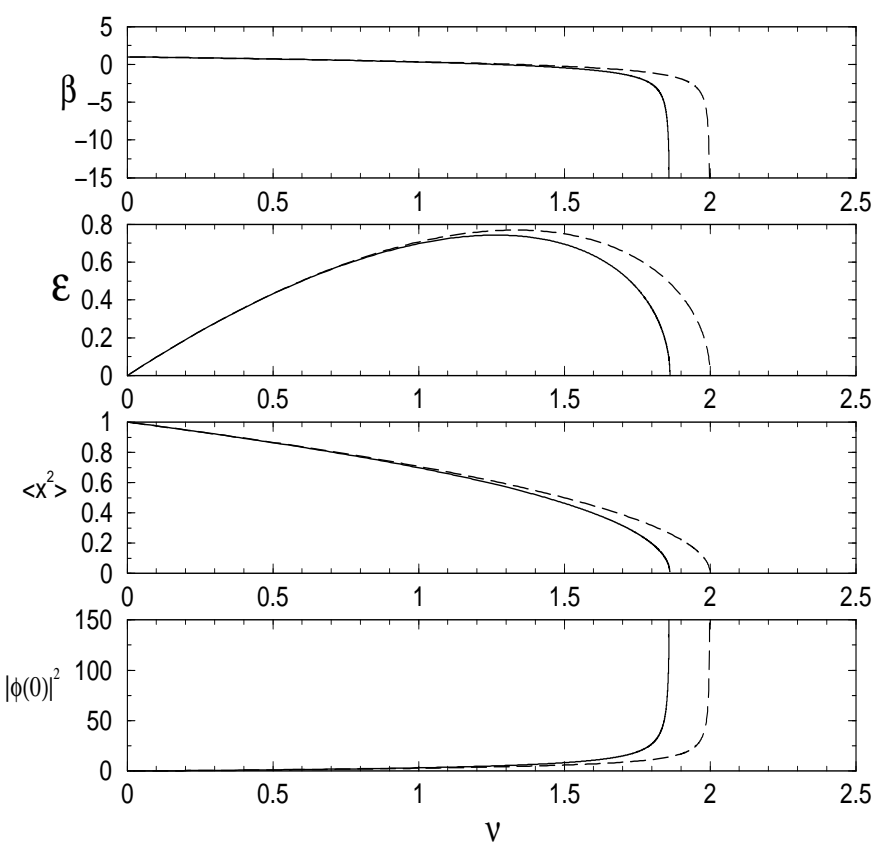

FIG. 2. For $D=2$, we present the variational and exact numerical calculations of the chemical potential $(\beta)$, total energy $(\mathcal{E})$, mean-square-radius $\left(\left\langle x^{2}\right\rangle\right)$, and central density $\left(|\phi(0)|^{2}\right)$, as a function of the reduced number of atoms $\nu$. All the quantities are in dimensionless units (see text). The solid line curves correspond to exact numerical results, while the dashed curves are the variational results.

Next, we also solve equation (10) exactly employing the shooting and Runge-Kutta methods, and compare the results with the corresponding variational ones. In this case, we consider only the particular interesting case of $D=2$, with $\lambda_{3}=0$. The results are shown in Fig. 2, for the chemical potential $\beta$, the total energy $\mathcal{E}$, meansquare-radius $\left\langle x^{2}\right\rangle$, and the central density $|\phi(0)|^{2}$. In order to numerically solve eq. (10), in the $s$-wave, we first write it in terms of the single variable $x \equiv \sqrt{\left(x_{1}^{2}+x_{2}^{2}\right)}$ and consider the following boundary conditions: $\phi^{\prime}(0)=$ 0 (where / stands for the derivative with respect to $x$ ) and $\phi(x) \rightarrow C \exp \left(-x^{2} / 2+[\beta-1] \ln (x)\right)$ when $x \rightarrow \infty$, where $C$ is a constant to be determined. As observed in Fig.2, the critical limit $\nu_{c}=2$ obtained analytically using the variational approach should be compared with $\nu_{c}=1.862$, obtained by exact numerical calculation. This critical limit was first obtained by Weinstein 10, in a non-linear approach with two-body term, without the trapping potential. The coincidence of the value with our exact calculation is due to the fact that at the critical limit the mean square radius goes to zero.

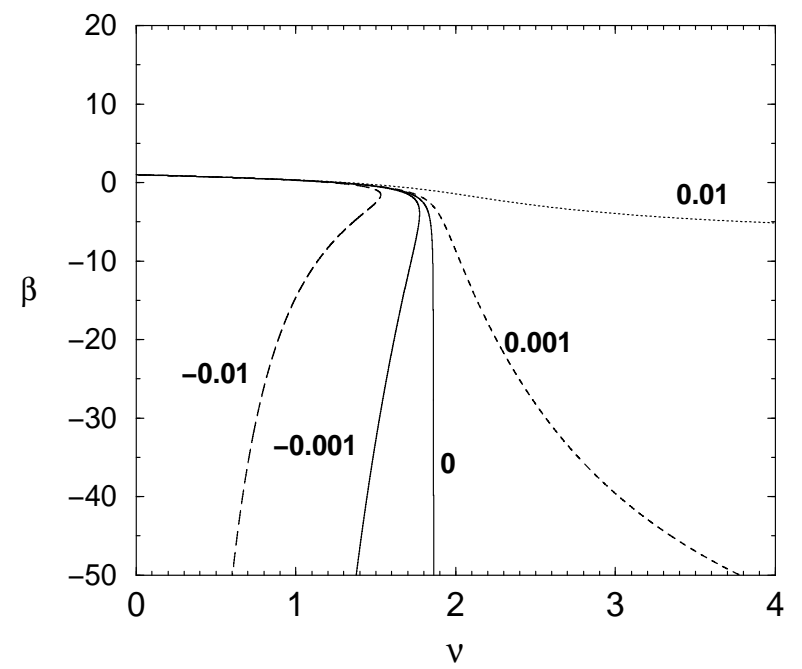

FIG. 3. Exact numerical solutions for $D=2$, of the chemical potential, $\beta$, in dimensionless units, given as function of the reduced number of atoms $\nu$, for different values of the three-body parameter $G_{3}$, when the space dimension is $D=2$. As shown, only when $G_{3} \leq 0$ the number of atoms is limited to certain critical number.

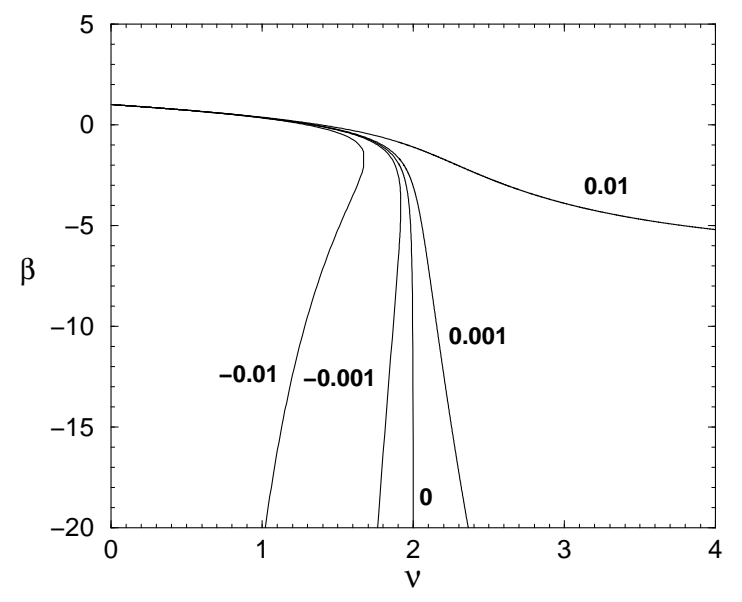

FIG. 4. The variational solutions for $D=2$, of the chemical potential, corresponding to the exact results given in Fig. 3 . 
We have also compared the results obtained by the variational approach with the exact numerical one, in the case of $D=2$, for several values of the three-body interaction term (positive and negative), as shown in Figs. 3 and 4 . In Fig. 3 we have the exact numerical approach and in Fig. 4 we have the corresponding variational results. By comparing the results we have for $D=2$ (shown in Figs. 3 and 4 ) with the ones obtained in ref. [5] for $D=3$, we should observe that no first order phase-transition exists in two dimensions. As observed in refs. [4, 6], for $D=3$, a first-order phase-transition can occur in trapped condensed states with negative two-body scattering length, when a repulsive three-body (quintic) term is added in the Hamiltonian. As shown in Figs. 3 and 4 , with $G_{3}$ positive the range of stability for the number of atoms $N$ can be increased indefinitely; with $G_{3}$ negative this range is reduced.

We can analyze the collapse conditions using "the virial theorem" approach 12. The mean square radius, $\left\langle r^{2}\right\rangle$, of a $D$-dimensional condensate, is given by

$$
\begin{aligned}
& \frac{d^{2}\left\langle r^{2}\right\rangle}{d t^{2}}+4 \omega^{2}\left\langle r^{2}\right\rangle= \\
& \frac{1}{m}\left[4\langle H\rangle+\lambda_{2}(D-2)\left\langle|\psi|^{2}\right\rangle+\frac{4 \lambda_{3}}{3}(D-1)\left\langle|\psi|^{4}\right\rangle\right],
\end{aligned}
$$

where

$$
\langle\mathcal{O}\rangle \equiv \frac{1}{N} \int d^{D} \vec{r} \psi^{\dagger}(\vec{r}, t) \mathcal{O} \psi(\vec{r}, t)
$$

and $\langle H\rangle=E / N$. When $\lambda_{3}=0$ we obtain the equation derived in 12 .

We can also write the eq. (13) in dimensionless units, as it was done in eqs. (810):

$$
\frac{d^{2}\left\langle x^{2}\right\rangle}{d \tau^{2}}+4\left\langle x^{2}\right\rangle=\frac{4 \mathcal{E}}{\nu}+2 f(\tau)
$$

where

$$
f(\tau) \equiv \frac{\lambda_{2}}{\left|\lambda_{2}\right|} \frac{D-2}{4}\left\langle|\phi|^{2}\right\rangle+G_{3} \frac{D-1}{3}\left\langle|\phi|^{4}\right\rangle .
$$

Using the initial conditions for $\left\langle x^{2}\right\rangle$ and $d\left\langle x^{2}\right\rangle / d \tau$, where, for simplicity, we assume $d\left\langle x^{2}\right\rangle / d \tau=0$, the solution of eq. (15) is given by

$$
\begin{aligned}
\left\langle x^{2}\right\rangle & =\frac{\mathcal{E}}{\nu}+\left[\left.\left\langle x^{2}\right\rangle\right|_{0}-\frac{\mathcal{E}}{\nu}\right] \cos (2 \tau) \\
& +\int_{0}^{\tau} f\left(\tau^{\prime}\right) \sin \left(2\left(\tau-\tau^{\prime}\right)\right) d \tau^{\prime}
\end{aligned}
$$

The stability regions and the estimates for the collapse time can be obtained from the analysis of this solution, like as performed for the case $\lambda_{3}=0$ in 15 . Let us analyze the dynamics when $D=2$. In this case, $\lambda_{2}$ does not appear explicitly in $\left\langle x^{2}\right\rangle$ and $f(\tau)$ also does not depend on this parameter:
1. For a positive $G_{3}$, negative $\lambda_{2}$ and $\mathcal{E}>0$ we observe that $\left\langle x^{2}\right\rangle$ cannot be zero and the condensate is stable. The mean square radius of the condensate oscillates in time around a finite value. This is confirmed by the numerical simulations (see Figs. 3 and 4$)$.

2 . For a negative $G_{3}$, positive $\lambda_{2}$ an analysis of stability like the one performed in ref. [15] shows that

a) When the total energy $\mathcal{E}<0$, the condensate is unstable and the wavefields collapse in a finite time at any initial conditions;

b) When $\mathcal{E}>0$, as the function $f(\tau)$ is negative, the contribution of the integral term for $\tau<\pi$ is negative. Then, we found the collapse condition as

$$
\left.\left\langle x^{2}\right\rangle\right|_{0} \geq 2 \frac{\mathcal{E}}{\nu}
$$

The same kind of analysis, for $D>2$, is much involved in the present approach, as the sign of the function $f(\tau)$ is not fixed at opposite signs for the parameters $\lambda_{2}$ and $\lambda_{3}$.

Some information about the dynamics of the collapse can also be obtained by using the techniques based on integral inequalities 10,16]. For instance, when $D=2$, we can estimate the three-body term contribution in $E$, following the procedure given in 10

$$
\int d^{2} \vec{r}|\psi|^{6} \leq C_{2}\left(\int d^{2} \vec{r} \frac{|\nabla \psi|^{2}}{2 m}\right)^{2}\left(\int d^{2} \vec{r}|\psi|^{2}\right)=C_{2} K^{2} N
$$

where $K$ is the kinetic energy and $C_{2}$ is defined from the minimization of the functional

$$
\mathcal{J}=\frac{\left(\int d^{2} \vec{r}|\nabla \psi|^{2}\right)^{2}\left(\int d^{2} \vec{r}|\psi|^{2}\right)}{\int d^{2} \vec{r}|\psi|^{6}}
$$

Combining with the corresponding estimate for $\int d^{2} \vec{r}|\psi|^{4}$, we obtain $E>E(K)$, where

$$
E(K)=K+\frac{\omega^{2} N^{2}}{4 K}+\frac{\lambda_{2}}{2} C_{1} N K+\frac{\lambda_{3}}{3} C_{2} K^{2} N .
$$

When $\lambda_{3}=0$ we get the equation derived in 12 . Equation (21) should be compared with the corresponding variational expression (5), where the kinetic energy is given by $K=N \hbar \omega /\left(2 \alpha^{2}\right)$ and $\alpha$ is the width of the cloud. As we see, the expression for the energy 21 is very similar to the obtained by the variational approach. However, (21) is valid for arbitrary time and describes the nonstationary dynamics. By using the variational expression (upper limit) for the ground-state, and the right-hand-side of eq. (21) (lower limit), we can approach analytically the exact solution for the total energy

$$
E(K)<E<E_{v a r}
$$


For a more deep insight to the problem of stability, we need to obtain the values of the constants $C_{1}$ and $C_{2}$. This problem requires a generalization of the method suggested by Weinstein in [10, to be considered in a future work.

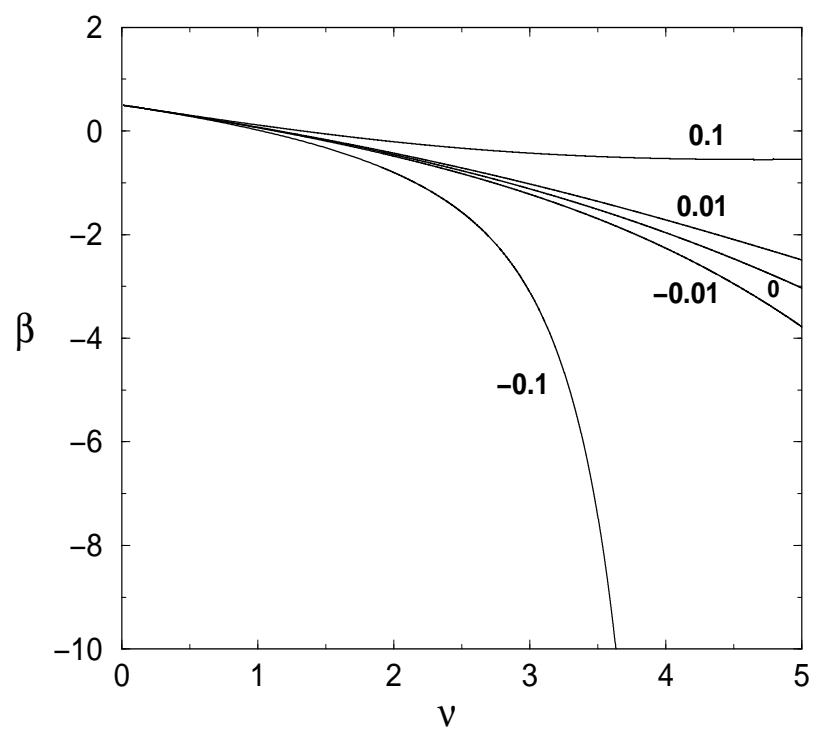

FIG. 5. Variational solutions for the chemical potential (in dimensionless units) as functions of $\nu$, for $D=1$ and different values of the three-body parameter $G_{3}$.

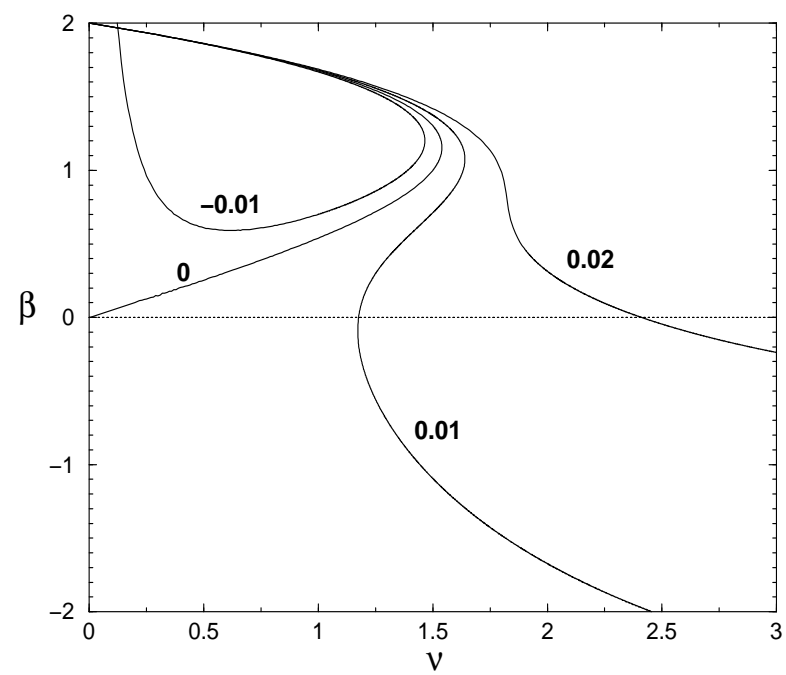

FIG. 6. The same as in Fig. 5, for $D=4$.

We should observe that exact numerical results, when $G_{3}=0$, have already been considered in refs. [14 (for $D=1$ and $D=3$ ), in [5] (for $D=3$ ), and in [17] (for $D=2$ ). In $[4]$, for $D=3$, it was also considered the case with $G_{3} \neq 0$, and shown a kind of first-order phasetransition in the condensate. In the present work, we have extended the variational formalism, in case $G_{3} \neq 0$, for an arbitrary $D$-dimension. In the following Figs. 5,
6 and 7 , we present our results for the chemical potential as a function of $\nu$, for a set of given values of $G_{3}$, in case of $D=1,4$ and 5. As one can observe in Fig. 5, even in case of $D=1$ one can reach a critical maximum limit for $\nu$, when $G_{3}$ is enough negative. For $D=4$ and 5 (Figs. 6 and 7 ), we observe similar picture of first-order phase-transition occurring for some specific values of $G_{3}$.

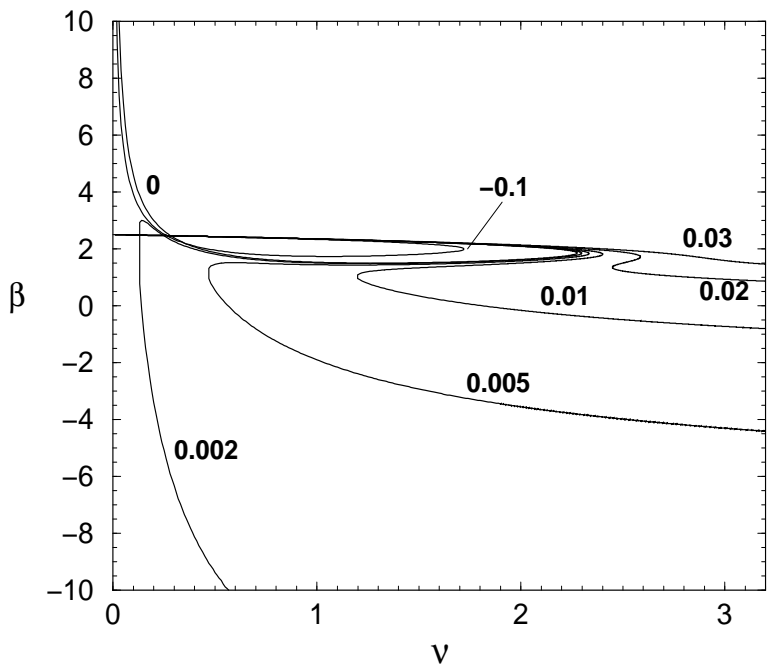

FIG. 7. The same as in Fig. 5 , for $D=5$.

In conclusion, in the present work, we first studied the stability and the critical number of atoms in arbitrary $D$-dimensions using a variational procedure, for the case we have two-body (attractive) and three-body contributions. This part extends a previous analysis done in refs. 11, 15. Next, we considered in more detail the case $D=2$.. We compared the variational results with exact numerical calculations for the chemical potential, total energy, mean-square-radius and density. Finally, we extended numerically the approach for $D=2$, including an effective three-body interaction term. We studied the sensibility of the critical numbers with respect to corrections in the non-linear interaction. The effective interaction considered in the equation contains a trapped harmonic interaction, and two nonlinear terms, proportional to the density $|\psi|^{2}$ (due to first-order two-body interaction) and to $|\psi|^{4}$ (due to first-order three-body interaction). We also verified, by a variational procedure, that a critical number of particles exists only for $D \geq 2$, when the nonlinear term of the NLSE contains just the cubic term. In case of $D=1$, a critical maximum number of atoms can exist with the addition of a negative quintic term (three-body) in the NLSE. In all cases where the number of atoms is limited, we observed that the addition of a positive $|\psi|^{4}$ allows stable solutions beyond the critical number. We also introduced an analysis of the collapse conditions, using "the virial theorem" approach given in 12]. The dynamics of the collapse was discussed in terms of the techniques developed in $[10$. In particular, we showed how the exact energy can be approached 
in the case of $D=2$ with two and three-body term contributions.

Acknowledgments We are grateful to Jordan M. Gerton for the suggestions and careful reading of the manuscript. This work was partially supported by Fundação de Amparo à Pesquisa do Estado de São Paulo and Conselho Nacional de Desenvolvimento Científico e Tecnológico.

[1] M.H. Anderson, J.R. Ensher, M.R. Matthews, C.E. Wieman, E.A. Cornell, Science 269 (1995) 198; C.C. Bradley, C.A. Sackett, J.J. Tollet, R.G. Hulet, Phys.Rev. Lett. 75 (1995) 1687; K.B. Davis, M.-O. Mewes, M.R. Andrews, N.J. van Druten, D.S.Durfee, D.M. Kurn, and W.Ketterle, Phys. Rev. Lett. 753969 (1995); D.G. Fried, T.C. Killian, L. Willmann, D. Landhuis, A.C. Moss, T.J. Greytak, and D. Kleppner, Phys. Rev. Lett. 81, 3811 (1998).

[2] V.L. Ginzburg and L.P. Pitaevskii, Zh. Eksp. Teor. Fiz. 34 (1958) 1240 [Sov. Phys. JETP 7 (1958) 858]; L.P. Pitaevskii, Sov. Phys. JETP 13 (1961) 451; E.P. Gross, J. Math. Phys. 4, 195 (1963).

[3] M. Wadati and T. Tsurumi, Phys. Lett. A 247 (1998) 287.
[4] N. Akhmediev, M.P. Das, and A.V. Vagov, in "Condensed Matter Theories", vol. 12, p. 17-25, edited by J.W. Clark and P.V. Panat (Nova Science Publ., New York, 1997).

[5] A. Gammal, T. Frederico, and L. Tomio, Phys. Rev. E 60 (1999) 2421; A. Gammal, T. Frederico, and L. Tomio, in "Collective Excitations in Fermi and Bose Systems", p. 159-168, edited by C. Bertulani, L.F. Canto and M. Hussein (World Scientific, Singapore, 1999).

[6] A. Gammal, T. Frederico, L. Tomio, and Ph. Chomaz, Liquid-Gas phase transition in Bose-Einstein Condensates with time evolution, submitted for publication.

[7] S.Inouye, M.R. Andrews, J. Stenger, H.J. Miesner, D.M. Stamper-Kurn, and W. Ketterle, Nature 392 (1998) 151.

[8] V. Efimov, Phys. Lett. B 33 (1970) 563.

[9] V.E. Zakharov, Sov. Phys. JETP 35 (1972) 908; V.E. Zakharov, V.S. Synakh, Sov. Phys. JETP 41 (1975) 465.

[10] M.I. Weinstein, Commun. Math. Phys. 87 (1983) 567.

[11] T. Tsurumi and M. Wadati, J. of Phys. Soc. of Japan 68 (1999) 1531; L. Bergé, T.J. Alexander, and Y.S. Kivshar, e-print: cond-mat/9907408.

[12] L.P. Pitaevskii, Phys.Lett. A 221 (1996) 14.

[13] G. Baym and C.J. Pethick, Phys. Rev. Lett. 76 (1996) 6.

[14] M. Edwards and K. Burnett, Phys. Rev. A 51 (1995) 1382; P.A. Ruprecht, M.J. Holland, K. Burnett, and M. Edwards, Phys. Rev. A 51 (1995) 4704.

[15] T.Tsurumi, H. Morise, and M. Wadati, e-print: condmat/9912470.

[16] S.K. Turitsyn, Phys.Rev. E 47 (1993) R13.

[17] S.K. Adhikari, Phys. Lett. A 265 (2000) 91. 